\title{
Erratum to: An integrative genomics approach for identifying novel functional consequences of PBRM1 truncated mutations in clear cell renal cell carcinoma (ccRCC)
}

\author{
Yuanyuan Wang ${ }^{1}$, Xingyi Guo ${ }^{1,2}$, Michael J. Bray ${ }^{3}$, Zhiyong Ding ${ }^{4}$ and Zhongming Zhao ${ }^{1,5,6,7^{*}}$
}

From The International Conference on Intelligent Biology and Medicine (ICIBM) Indianapolis, IN, USA. 13-15 November 2015

\section{Erratum}

Following publication of the original article [1] it was brought to our attention that there were three errors in the "Results" section of the article's abstract. Please see below for the two sentences in which these errors occur:

1. We identified 613 differentially expressed genes (128 up-regulated and 485 down-regulated genes using cutoff $\left|\log _{2} \mathrm{FC}\right|<1$ and $\left.p<0.05\right)$ in PBRM1 mutated group versus "pan-negative" group.

2. In addition, we identified 1405 differentially methylated CpG sites (targeting 1308 genes, $\left|\log _{2} \mathrm{FC}\right|<1, p<0.01$ ) and 185 significantly altered microRNAs $\left(\left|\log _{2} \mathrm{FC}\right|<1, p<0.05\right)$ associated with truncated PBRM1 mutations.

In both sentences the present expression in the article is " $\left|\log _{2} \mathrm{FC}\right|<1$ ". However, this is incorrect, as the cutoff should be " $\left|\log _{2} \mathrm{FC}\right|>1 "$.

\section{Author details}

'Department of Biomedical Informatics, Vanderbilt University School of Medicine, Nashville, TN 37203, USA. ²Division of Epidemiology, Department of Medicine, Vanderbilt University School of Medicine, Nashville, TN 37232, USA. ${ }^{3}$ Vanderbilt Genetics Institute, Vanderbilt University School of Medicine, Nashville, TN 37232, USA. ${ }^{4}$ Department of Systems Biology, University of Texas MD Anderson Cancer Center, Houston, TX 77030, USA. ${ }^{5}$ Department of Cancer Biology, Vanderbilt University School of Medicine, Nashville, TN 37232, USA. ${ }^{6}$ Department of Psychiatry, Vanderbilt University School of Medicine, Nashville, TN 37212, USA. ${ }^{7}$ Center for Precision Health, School of
Biomedical Informatics, The University of Texas Health Science Center at Houston, Houston, TX 77030, USA.

Received: 28 September 2016 Accepted: 28 September 2016 Published online: 21 October 2016

\section{References}

1. Wang $Y$, et al. An integrative genomics approach for identifying novel functional consequences of PBRM1 truncated mutations in clear cell renal cell carcinoma (ccRCC). BMC Genomics. 2016;17 Suppl 7:515.

*Correspondence: zhongming.zhao@uth.tmc.edu

From The International Conference on Intelligent Biology and Medicine (ICIBM), Indianapolis, IN, USA. 13-15 November 2015

'Department of Biomedical Informatics, Vanderbilt University School of Medicine, Nashville, TN 37203, USA

${ }^{5}$ Department of Cancer Biology, Vanderbilt University School of Medicine, Nashville, TN 37232, USA 